\title{
Efeito de imunodulador glutamina e probiótico pool de Lactobacillus na atenuação dos sintomas e crises de asma em crianças
}

\author{
Effect of the glutamine and Lactobacillus pool on the attenuation of clinical asthma symptoms \\ and attacks in children
}

\section{DOI: $10.37111 /$ braspenj.2019344007}

Michele Honicky'

João Paulo Zattar ${ }^{2}$

Mariana Saciloto ${ }^{3}$

Mariana Abe Vicente Cavagnari ${ }^{4}$

Claudia Nodari ${ }^{5}$

Angelica Rocha de Freitas Melhem ${ }^{6}$

\section{Unitermos:}

Criança. Doenças respiratórias. Fatores imunológicos. Glutamina. Probióticos. Lactobacillus.

\section{Keywords:}

Child. Respiratory tract diseases. Immunologic factors. Glutamine. Probiotics. Lactobacillus.

\section{Endereço para correspondência:}

Michele Honicky

Rua José Weiss, 270 - Boehmerwald - São Bento do Sul, SC, Brasil - CEP 89287-630.

E-mail: michele_honicky@yahoo.com.br

\section{Submissão}

13 de agosto de 2019

\section{Aceito para publicação}

27 de outubro de 2019

\begin{abstract}
RESUMO
Introdução: Imunomodulador glutamina e probiótico Lactobacillus, em determinadas doses, têm efeitos benéficos, pela modulação do sistema imunológico, podendo auxiliar na integridade do sistema respiratório. Entretanto, estudos sobre os efeitos da glutamina ou pool de Lactobacillus na prevenção e controle da asma em crianças ainda são escassos. O objetivo do estudo foi avaliar efeito do uso da glutamina e pool de Lactobacillus na atenuação de sintomas clínicos de asma em crianças. Método: Estudo longitudinal, 45 crianças com diagnóstico médico de asma distribuídos em três grupos: grupo controle (C), grupo glutamina (G), grupo Lactobacillus (L). O grupo $G$ foi suplementado com L-glutamina em pó $(0,3 \mathrm{~g} / \mathrm{kg} / \mathrm{dia})$. O grupo L utilizou um pool de Lactobacillus (Lactobacillus casei, paracasei, rhamnosus, acidophilus e Bifidobacterium lactis) $(2 \mathrm{~g} / \mathrm{dia}) .0$ grupo $C$ não recebeu glutamina nem pool de Lactobacillus. Foram avaliadas as características clínicas e sintomas das doenças respiratórias por meio de anamnese específica para o estudo, e aplicado o Questionário ISSAC (para obtenção de pontuações para diagnóstico e sintomas de asma), durante 4 meses. Para testar as diferenças entre os grupos, foi utilizado o teste ANOVA, com post-hoc de Tukey. Foi considerado significativo $p<0,05$. Resultados: Pontuação inicial para - ISSAC foi de $C=8,67 \pm 1,77, L=7,80 \pm 1,52$ e $G=8,00 \pm 1,46, p=0,31$. No primeiro acompanhamento, a pontuação do ISSAC indicou que o grupo $G$ teve melhora nas características clínicas de asma $(C=6,47 \pm 2,29, L=5,07 \pm 2,28, G=4,00 \pm 1,73, p<0,05)$, como também ocorreu nos meses seguintes até o último acompanhamento $(C=5,93 \pm 2,28, L=5,13 \pm 2,13, G=4,00 \pm 1,96, p<0,05)$. Após suplementação, o grupo $\mathrm{G}$ apresentou menor média de duração de crises de asma $(p=0,01)$, menor quantidade de crises de asma $(p<0,05)$, menor prevalência de sintomas típicos de asma: tosse e sibilos $(p<0,05)$. Conclusão: Suplementação de glutamina atenuou os sintomas típicos de asma, enquanto a utilização do pool de Lactobacillus não apresentou atenuação dos sintomas. Glutamina pode ser uma nova estratégia para prevenção e controle da asma em crianças.
\end{abstract}

\section{ABSTRACT}

Introduction: Immunomodulator glutamine and probiotic Lactobacillus at certain doses have beneficial effects by modulation of the immune system and may assist in the integrity of the respiratory system. However, studies about the effects of glutamine or Lactobacillus pool on the prevention and control of asthma in children are still scarce. The aim of study was to evaluate the effect of the use of glutamine and Lactobacillus pool on the attenuation of clinical asthma symptoms in children. Methods: Longitudinal study, 45 children with medical diagnosis of asthma distributed in three groups: control group $(C)$, glutamine group (G), Lactobacillus group (L). Group $G$ was supplemented with $L$-glutamine powder $(0.3 \mathrm{~g} / \mathrm{kg} / \mathrm{day})$. Group $L$ used a pool of Lactobacillus (Lactobacillus casei, paracasei, rhamnosus, acidophilus and Bifidobacterium lactis) $(2 \mathrm{~g} /$ day). Group C received no glutamine or Lactobacillus pool. Clinical characteristics and symptoms of respiratory diseases were assessed by study-specific anamnesis and ISSAC Questionnaire (to obtain diagnostic scores and asthma symptoms) for 4 months. To test for differences between groups, the ANOVA test with Tukey post-hoc test was used. It was considered significant $p<0.05$. Results: Initial ISSAC score was ( $C=8.67 \pm 1.77, L=7.80 \pm 1.52$ and $G=8.00 \pm 1.46, p=0.31)$. At the first follow-up, the ISSAC score indicated that group $\mathrm{G}$ had improvement in the clinical characteristics of asthma $(C=6.47 \pm 2.29, L=5.07 \pm 2.28, G=4.00 \pm 1.73, p<0.05)$, as also occurred in the following months until the last follow-up $(C=5.93 \pm 2.28, L=5.13 \pm 2.13, G=4.00 \pm 1.96, p<0.05)$ After supplementation, group $G$ presented lower mean duration of asthma attack $(p=0.01)$, lower number of asthma attack ( $<<0.05)$, lower prevalence of typical asthma symptoms, as cough and wheezing $(\mathrm{p}<0.05)$. Conclusion: Glutamine supplementation attenuated the typical asthma symptoms, while the use of Lactobacillus pool did not attenuate the symptoms. Glutamine may be a new strategy for prevention and control of asthma in children.

1. Mestre em Nutrição, Doutoranda em Nutrição no Programa de Pós-graduação em Nutrição pela Universidade Federal de Santa Catarina, Florianópolis, SC, Brasil.

2. Graduado em Medicina, Pediatra e Neonatologista na Clínica Zattar, Guarapuava, PR, Brasil.

3. Graduada em Medicina, Pediatra e Pneumologista Pediátrica na Bambina Clínica de Doenças Infantis, Guarapuava, PR, Brasil.

4. Doutora em Ciências (Gastroenterologia), Docente na Universidade Estadual do Centro-Oeste, Guarapuava, PR, Brasil.

5. Graduada em Enfermagem, Enfermeira na Clínica Zattar, Guarapuava, PR, Brasil.

6. Doutora em Ciências (Gastroenterologia), Docente na Universidade Estadual do Centro-Oeste, Guarapuava, PR, Brasil. 


\section{INTRODUÇÃO}

A asma é uma doença respiratória que acomete, aproximadamente, 300 milhões de pessoas mundialmente, principalmente as crianças. $\bigcirc$ Brasil encontra-se entre os países com maior prevalência de asma em crianças'.

Vários fatores podem influenciar no desenvolvimento das doenças respiratórias na infância, como fatores ambientais, exposição a polventes atmosféricos, tabagismo, animais de estimação, estresse, não realização do aleitamento materno, fatores genéticos, infecções virais das vias respiratórias, aspectos de higiene e uso de antibióticos ${ }^{2}$. No entanto, a ocorrência da asma é resultado de interações entre fatores genéticos e ambiente, que desencadeiam respostas anormais do sistema imunológico, gerando inflamação e hiper-reatividade das vias aéreas inferiores e obstrução do fluxo aéreo ${ }^{3}$.

Apesar da mortalidade infantil por asma ser baixa, o escasso controle e prevenção dos sintomas das doenças respiratórias aumenta a taxa de hospitalização, o que representa alto custo econômico ${ }^{4}$, bem como consequências no estado de saúde da criança, que podem afetar a qualidade de vida, rendimento escolar e baixa produtividade dos pais ${ }^{5}$.

Ao considerar que reações imunológicas têm papel no desenvolvimento da asma, nutrientes que possam modular o sistema imunológico poderiam auxiliar no controle da doença. A glutamina é um imunomodulador que, em determinadas doses, tem efeitos benéficos no sistema imune, por meio da modulação desse sistema, que resulta na diminuição das infecções, redução do tempo de internação, melhora da resposta inflamatória, integridade da mucosa intestinal e do sistema imune em crianças com doenças graves ${ }^{6}$. Os probióticos, especialmente os Lactobacillus, também podem melhorar a resposta imune quando administrados em quantidades adequadas, pois promovem o equilíbrio da microbiota intestinal e conferem benefícios à saúde do hospedeiro. Possuem efeitos benéficos principalmente na função intestinal, porém também podem aumentar as imunoglobulinas com ação anti-inflamatórias ${ }^{7}$.

Frente a esses fatores, glutamina e Lactobacillus, por terem possíveis efeitos benéficos no sistema imune, poderiam apresentar também ação no sistema respiratório, atuando como uma nova estratégia para o controle da asma. No entanto, estudos que avaliam os efeitos dos imunomoduladores e probióticos na prevenção e no controle da asma em crianças ainda são escassos.

Nesse contexto, o objetivo deste estudo foi avaliar o efeito do uso do imunomodulador glutamina e do probiótico Lactobacillus na atenuação de sintomas clínicos de asma, em crianças menores de sete anos de idade.

\section{MÉTODO}

Tratou-se de estudo longitudinal, realizado em Guarapuava-PR, Brasil, no período de março a setembro de 2015, com crianças menores de sete anos de idade, de ambos os gêneros, com diagnóstico médico prévio de asma, cujos pais concordaram voluntariamente que seu (sua) filho (a) participasse do estudo, assinando o Termo de Consentimento Livre e Esclarecido. O estudo foi aprovado no Comitê de Ética em Pesquisa (COMEP) da Universidade Estadual do CentroOeste (UNICENTRO), sob o parecer de número 1.142.314.

A amostra por conveniência foi composta por 45 crianças com asma, divididas em três grupos de forma aleatória. $\bigcirc$ grupo caso 1 foi suplementado com o imunomodulador L-glutamina em pó sem sabor, na quantidade de $0,3 \mathrm{~g} / \mathrm{kg} /$ dia $^{6}$, durante quatro meses. $\bigcirc$ grupo caso 2 utilizou probiótico, na forma de um pool de Lactobacillus (Lactobacillus casei $10^{8}$ UFC, Lactobacillus paracasei $10^{8}$ UFC, Lactobacillus rhamnosus $10^{8}$ UFC, Lactobacillus acidophilus $10^{8}$ UFC e Bifidobacterium lactis $10^{8}$ UFC) no excipiente iogurte, na quantidade de $2 \mathrm{~g} / \mathrm{dia}^{7}$, durante quatro meses. $\bigcirc$ grupo controle não recebeu imunomodulador nem probiótico. Nenhum grupo recebeu informação para mudança na alimentação. Todos os pais ou responsáveis dos grupos casos 1 e 2 receberam informações padronizadas sobre como inserir o imunomodulador ou probiótico na alimentação dos(as) filhos(as). A entrega do imunomodulador ou probiótico foi realizada mensalmente, e estes eram divididos em sachê para o uso diário, de acordo com a quantidade recomendada para cada criança.

A administração de medicamentos para todos os grupos foi mantida durante o estudo, de acordo com a prescrição médica, apresentando variações entre classes de medicamentos, doses e tempo de administração entre os participantes, sendo que estas informações foram avaliadas por meio de anamnese específica para estudo.

Os dados foram coletados por único avaliador, em cinco visitas previamente agendadas no domicílio do participante, por meio de questionários e avaliação nutricional. Na primeira e última visita, avaliou-se a prevalência das características clínicas da asma e realizou-se avaliação nutricional, com duração de aproximadamente 45 minutos. Mensalmente, verificou-se a implementação do uso de imunomodulador e probiótico, informações sobre a utilização, dificuldades e possíveis efeitos colaterais destes, por meio de questionário próprio, no domicílio do participante, bem como aspectos clínicos da asma e estado nutricional das crianças.

Para a avaliação das características clínicas e dos sintomas da asma foi aplicada anamnese específica para o estudo e o Questionário ISAAC - Módulo Asma do Questionário Padrão Internacional (The Internacional Study of Asthma and Allergies in Childhood - ISAAC). O questionário é uma ferramenta para 
diagnóstico de asma e alergias em estudos epidemiológicos. De acordo com o objetivo do estudo, foi aplicado apenas o módulo da asma do ISAAC, que avalia ocorrência de sintomas da asma, gravidade dos sintomas e diagnóstico da asma por meio de perguntas simples e cada resposta corresponde a um escore, sendo que o escore global do ISAAC igual ou maior a cinco pontos, indica diagnóstico de asma e alergias respiratórias em crianças ${ }^{8}$. Os pais ou responsável pelo participante também eram questionados sobre ano do diagnóstico da asma, duração das crises, intervalo das crises, sintomas e os episódios e frequência de internamento hospitalar.

Realizou-se avaliação antropométrica para obtenção do peso $(\mathrm{kg})$ e estatura $(\mathrm{m})$, seguindo os protocolos específicos para aferição das medidas de crianças. $\bigcirc$ peso foi obtido em balança digital portátil, com precisão de 100 gramas e capacidade para $150 \mathrm{~kg}$. A estatura foi determinada verticalmente com utilização de fita métrica fixada em parede sem rodapé. A classificação e o diagnóstico do estado nutricional foram obtidos por meio dos índices Peso/ldade (P/I), Estatura/ldade (E/I) e Índice de Massa Corporal/Idade (IMC/I), utilizados os valores de referência de escore-z para crianças de zero a dez anos de idade, propostos pela Organização Mundial da Saúde" com o auxílio do módulo antropométrico WHO Anthro ${ }^{\circledR}$ versão 3.2.2.

Os dados coletados foram organizados em planilhas, com auxílio do software Excel $\circledast$. Inicialmente, os dados foram analisados por meio estatística descritiva, com médias, desvio padrão, frequências relativas e absolutas. Testes de normalidade (Shapiro-Wilk e Kolmogorov-Smirnov) foram utilizados para verificação da distribuição das variáveis numéricas. A comparação das variáveis numéricas foi realizada por meio do teste-t de Student para amostras independentes e por meio da Análise de Variância (ANOVA) e teste post-hoc de Tukey. A comparação das variáveis categóricas foi realizada por meio do teste do Qui-Quadrado. Todas as análises foram realizadas com auxílio do Software SPSS versão 20.0, com nível de significância de $5 \%(p<0,05)$.

\section{RESULTADOS}

Quarenta e cinco crianças com diagnóstico médico prévio de asma participaram do estudo, com média de idade de $3,69 \pm 1,58$ anos. Destas, $62,2 \%(n=28)$ eram do sexo masculino. A média de idade do diagnóstico médico de asma foi 1,5 $\pm 0,9$ anos e verificada alta prevalência de histórico familiar de doença respiratória $(86,7 \%, n=39)$. Algumas crianças apresentaram peso ao nascer insuficiente $(35,6 \%, n=16)$ e baixo peso $(4,4 \%, n=2)$, porém com maior predomínio de idade gestacional a termo $(86,7 \%, n=39)$. As crianças apresentam crises de asma com duração média de $9,3 \pm 4,4$ dias, com intervalos entre as crises variando de apenas uma semana até quatro meses ou mais.
Os sintomas mais frequentes nas crises foram tosse $(91,1 \%, n=41)$, sibilo $(93,3 \%, n=42)$, sono alterado $(68,9 \%$, $n=31)$, coriza $(66,7 \%, n=30)$, dispneia $(64,4 \%, n=29)$ e febre $(37,8 \%, n=17)$. Dentre as crianças avaliadas, $42,2 \%$ $(n=19)$ não tinham histórico de internamento e $46,7 \%$ $(n=21)$ apresentavam internamentos esporádicos ao longo de um ano.

No início do estudo, houve administração em todos os grupos de anti-histamínicos (controle $20 \% \mathrm{n}=3$; Lactobacillus $26,6 \% n=4$; glutamina $13,3 \% n=2$ ), antibióticos (controle 13,3\% $n=2$; Lactobacillus 6,6\% $n=1$; glutamina $0 \% \mathrm{n}=0$ ) e corticoides (controle 13,3\% $\mathrm{n}=2$; Lactobacillus $26,6 \% n=4$; glutamina $6,6 \% n=1$ ), sem diferença significativa entre os grupos $(p>0,05)$. Apenas a administração de broncodilatador (controle 33,3\% $n=5$; Lactobacillus $93,3 \% n=14$; glutamina $73,3 \% n=11$ ) foi maior no grupo dos Lactobacillus $(p=0,002)$ e antiasmáticos (controle 46,6\% n=7; Lactobacillus 46,6\% n=7); glutamina 86,6\% $n=13)$ no grupo da glutamina $(p=0,036)$. Ao longo dos quatro meses de estudo, verificou-se maior prevalência de administração de broncodilatadores $(p=0,054)$, no primeiro acompanhamento, antibiótico $(p=0,012)$, no segundo acompanhamento, no grupo controle. O grupo da glutamina apresentou menor administração de corticoides $(p=0,022)$, no terceiro acompanhamento. As demais classes de medicamentos não apresentaram diferença significativa entre os grupos.

Em relação ao estado nutricional das crianças no decorrer do estudo, observou-se aumento de peso e estatura em todos os grupos, o que era esperado pela evolução natural do crescimento e desenvolvimento das crianças. Ressalta-se que as crianças não receberam intervenção nutricional no estudo, entretanto podem ter sido orientadas por pediatra e/ou nutricionista. Os três grupos apresentaram diferença significativa na classificação do IMC/I após os quatro meses de estudo (Tabela 1).

Por meio do escore do Questionário ISAAC verificou-se distribuição homogênea dos grupos no início do estudo, o que permitiu a comparação entre eles para verificar a atenuação dos sintomas ao longo dos quatro meses.

O grupo que recebeu glutamina apresentou melhora clínica dos sintomas de asma, considerando o escore do ISAAC em todos os meses de suplementação, com a menor média do escore. Além disso, o grupo da glutamina apresentou escore do ISAAC menor que cinco ao longo da suplementação, que indica a ausência de diagnóstico de asma e alergias respiratórias em crianças. No entanto, ressalta-se que estas crianças apresentam diagnóstico clínico de asma, e o que ocorreu foi uma melhora dos sintomas clínicos da doença, resultando na diminuição do escore de ISAAC ao longo do estudo (Figura 1). 
Tabela 1 - Estado nutricional de crianças com asma por grupo: controle, Lactobacillus e glutamina.

\begin{tabular}{|c|c|c|c|c|c|c|c|c|c|c|}
\hline & & \multicolumn{3}{|c|}{ Controle } & \multicolumn{3}{|c|}{ Lactobacillus } & \multicolumn{3}{|c|}{ Glutamina } \\
\hline & & Inicial & Final & $p$ & Inicial & Final & $p$ & Inicial & Final & $p$ \\
\hline \multicolumn{2}{|c|}{ Peso média $( \pm \mathrm{DP}) \mathrm{kg}$} & $\begin{array}{l}19,71 \\
(7,62)\end{array}$ & $\begin{array}{l}20,74 \\
(7,85)\end{array}$ & 0,001 & $\begin{array}{l}18,88 \\
(5,073)\end{array}$ & $\begin{array}{c}20,06 \\
(5,489)\end{array}$ & 0,004 & $\begin{array}{c}16,80 \\
(3,678)\end{array}$ & $\begin{array}{c}17,74 \\
(3,742)\end{array}$ & $<0,001$ \\
\hline \multicolumn{2}{|c|}{ Estatura média $( \pm D P)$ m } & $\begin{array}{l}1,06 \\
(0,15)\end{array}$ & $\begin{array}{l}1,07 \\
(0,15)\end{array}$ & $<0,001$ & $\begin{array}{c}1,04 \\
(0,144)\end{array}$ & $\begin{array}{c}1,07 \\
(0,136)\end{array}$ & $<0,001$ & $\begin{array}{c}1,02 \\
(0,11)\end{array}$ & $\begin{array}{c}1,05 \\
(0,107)\end{array}$ & $<0,001$ \\
\hline \multirow{4}{*}{$\begin{array}{l}\mathrm{P} / \mathrm{l} \\
\text { (escore z) } \\
\% \text { (n) }\end{array}$} & $\begin{array}{c}\text { Muito baixo peso para } \\
\text { idade }\end{array}$ & - & - & & - & - & & - & - & - \\
\hline & Baixo peso para idade & - & - & & - & - & & - & - & \\
\hline & $\begin{array}{c}\text { Peso adequado para } \\
\text { idade }\end{array}$ & $80(12)$ & $80(12)$ & NA & $60(9)$ & $73,3(11)$ & 0,004 & $100(15)$ & $100(15)$ & \\
\hline & $\begin{array}{c}\text { Peso elevado para } \\
\text { idade }\end{array}$ & $20(3)$ & $20(3)$ & & $40(6)$ & $26,7(4)$ & & - & - & \\
\hline \multirow{3}{*}{$\begin{array}{l}\text { E/I } \\
\text { (escore z) } \\
\%(n)\end{array}$} & $\begin{array}{l}\text { Muita baixa estatura } \\
\text { para idade }\end{array}$ & - & - & & - & - & & - & - & \\
\hline & $\begin{array}{l}\text { Baixa estatura para } \\
\text { idade }\end{array}$ & $6,7(1)$ & - & NA & - & - & NA & - & - & NA \\
\hline & $\begin{array}{c}\text { Estatura adequada } \\
\text { para idade }\end{array}$ & $93,3(14)$ & $100(15)$ & & $100(15)$ & $100(15)$ & & $100(15)$ & $100(15)$ & \\
\hline \multirow{7}{*}{$\begin{array}{l}\text { MC/I } \\
\text { (escore z) } \\
\%(n)\end{array}$} & Magreza acentuada & - & - & & - & - & & - & - & \\
\hline & (escore z) & - & - & & - & - & & - & - & \\
\hline & $\%(n)$ & $66,7(10)$ & $53,3(8)$ & & $53,3(8)$ & $53,3(8)$ & & $73,3(11)$ & $80(12)$ & \\
\hline & Risco de sobrepeso & $13,3(2)$ & $13,3(2)$ & 0,008 & $13,3(2)$ & - & 0,046 & $20(3)$ & $13,3(2)$ & $<0,001$ \\
\hline & Sobrepeso & $6,7(1)$ & $13,3(2)$ & & $33,3(5)$ & $40,0(6)$ & & $6,7(1)$ & 6,7 (1)) & \\
\hline & Obesidade & $6,7(1)$ & $13,3(2)$ & & - & $6,7(1)$ & & - & - & \\
\hline & Obesidade grave & $6,7(1)$ & $6,7(1)$ & & - & - & & - & - & \\
\hline
\end{tabular}

Notas: P/l: Peso/ Idade E/l: Estatura/ldade IMC/I: Índice de massa corporal/Idade

$\%=$ porcentagem; $n=$ número amostral; $p=$ valor estatístico do teste; $N A=$ não se aplica; $D P=$ desvio padrão.

Tabela 2 - Comparação dos números de crises, duração das crises e internamentos de crianças com asma por grupo: controle, Lactobacillus e glutamina.

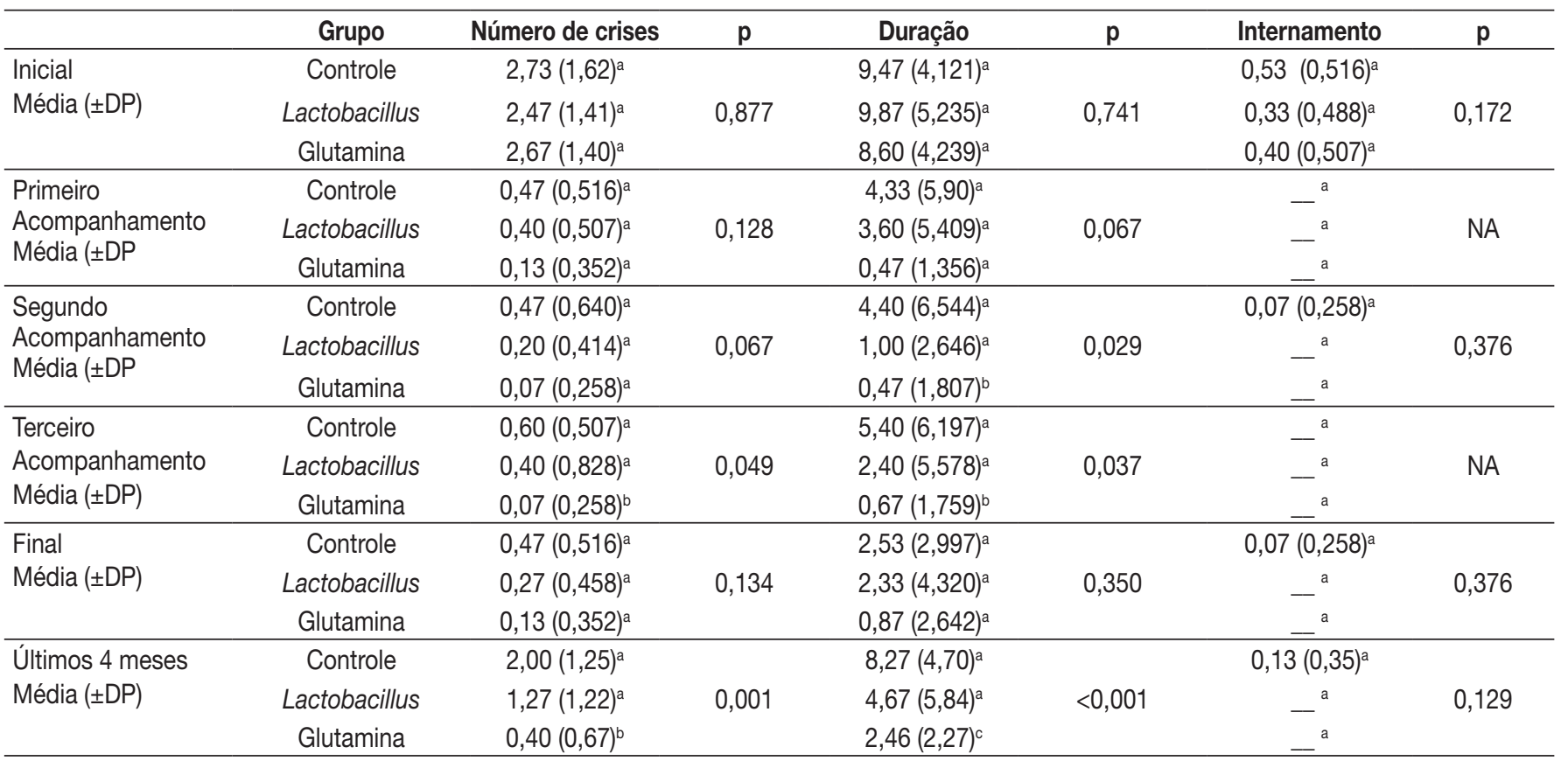

Notas: $p=$ valor estatístico do teste (ANOVA) DP= desvio padrão; NA= Não se aplica

${ }^{a}$ não apresentou diferença significativa dos demais grupos com valor de $p>0,05$ (Tukey)

${ }^{\mathrm{b}}$ apresentou diferença significativa do grupo controle com valor de $p<0,05$ (Tukey)

${ }^{c}$ apresentou diferença significativa do grupo controle com valor de $p=0,00$ (Tukey) 


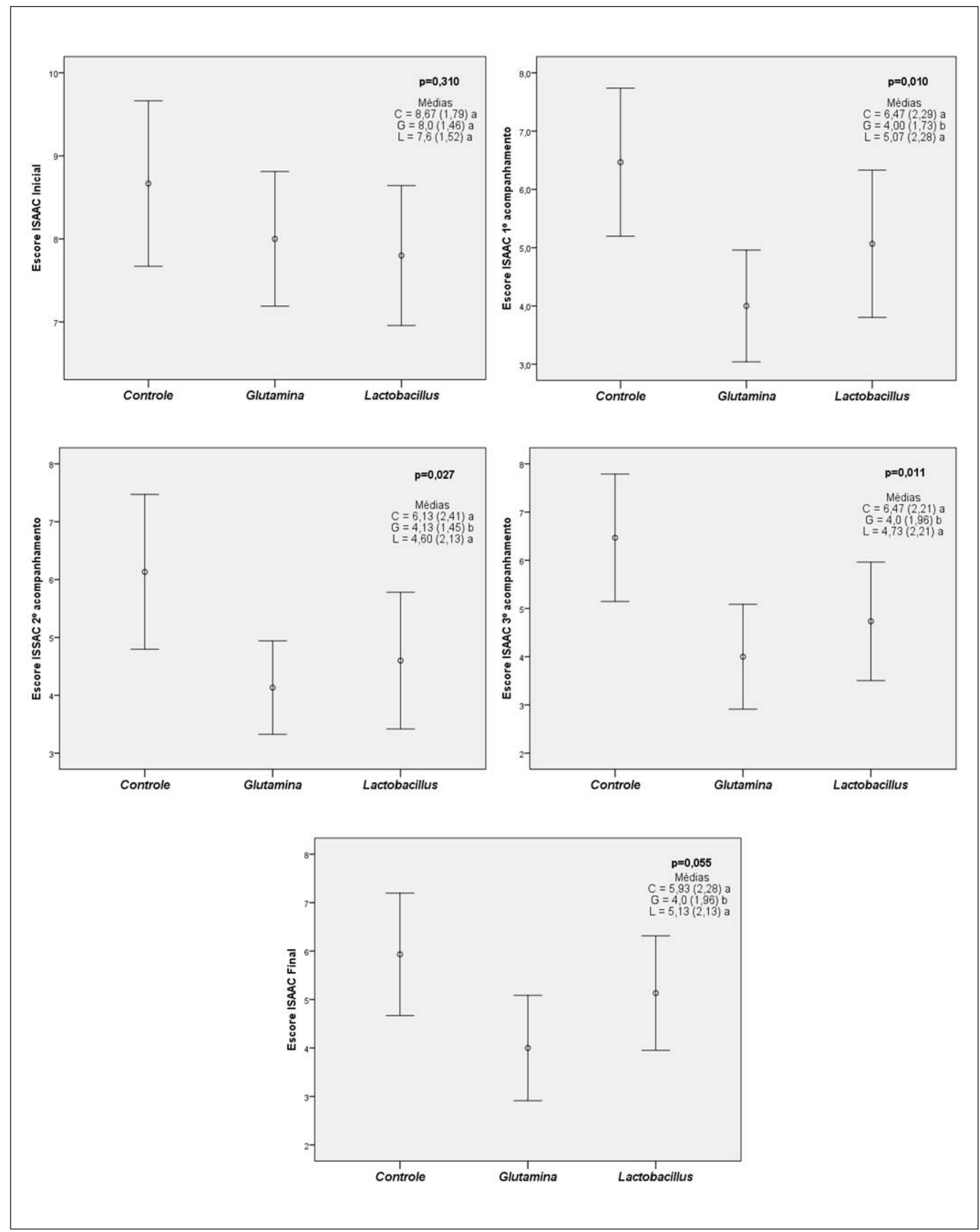

Figura 1 - Comparação do escore de ISAAC por grupo: controle, Lactobacillus e glutamina na verificação da atenuação de sintomas da asma em crianças. $C=$ Controle; $G=$ Glutamina; $L=$ Lactobacillus; $p=$ valor estatistico do teste (ANOVA)

${ }^{a}$ não apresentou diferença significativa dos demais grupos com valor de $p>0,05$ (Tukey).

${ }^{b}$ apresentou diferença significativa do grupo controle com valor de $p<0,05$ (Tukey). 

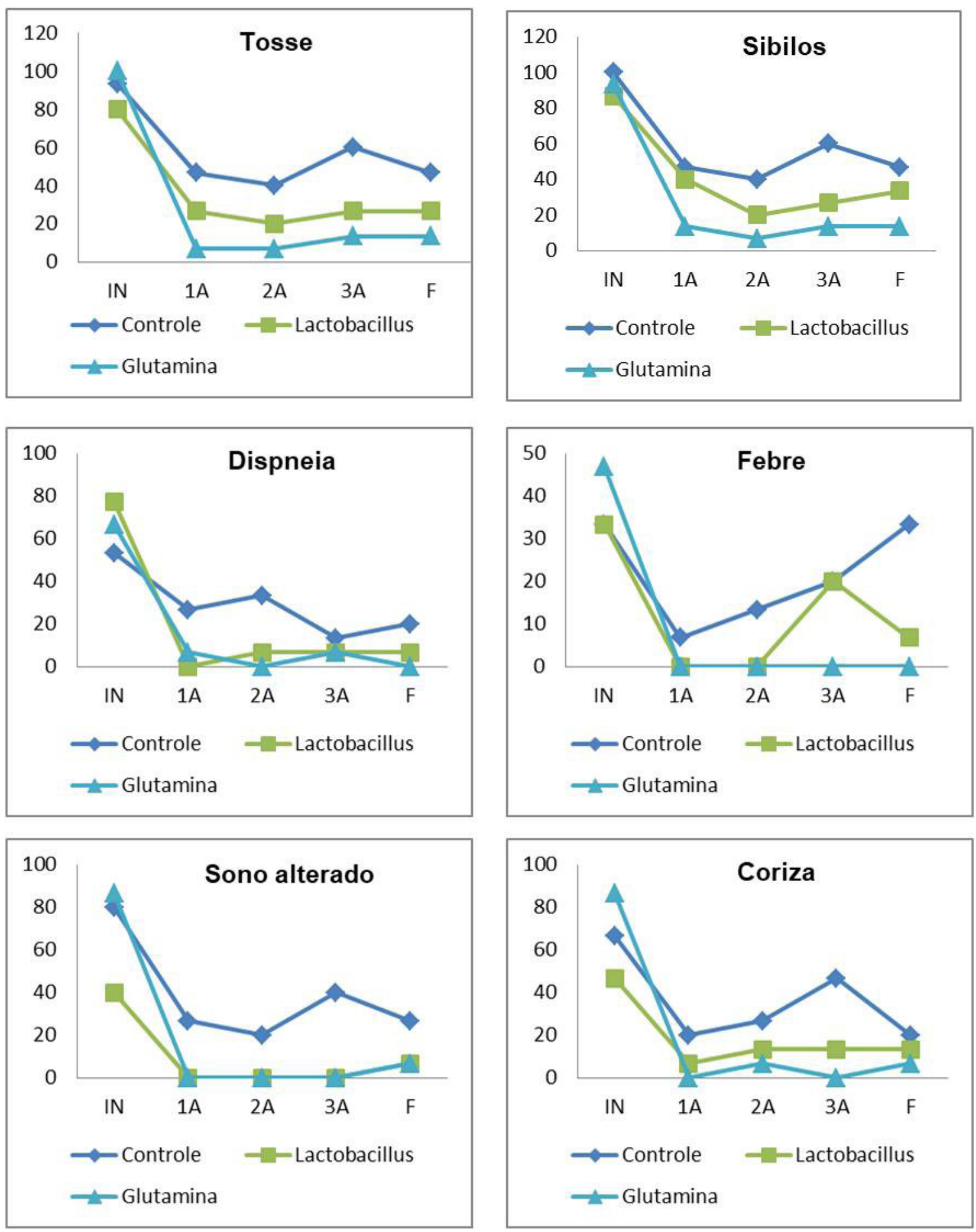

Figura 2 - Comparação dos sintomas clínicos da asma em crianças por grupo: controle, Lactobacillus e glutamina. Notas: Valores em percentual; $p=$ valor estatístico do teste;

IN: Inicial: 1A: Primeiro acompanhamento: 2A: Segundo acompanhamento; $3 \mathrm{~A}$ : Terceiro acompanhamento: F: Final.

IN: Tosse: $p=0,146$; Sibilos: $p=0,346$; Dispneia $p=0,507 ;$ Febre: $p=0,685$; Sono alterado: $p=0,012 ;$ Coriza: $p=0,067$

1A: Tosse: $p=0,047 ;$ Sibilos: $p=0,122 ;$ Dispneia: $p=0,054 ;$ Febre: $p=0,360 ;$ Sono alterado: $p=0,012 ;$ Coriza: $p=0,146$

2A: Tosse: $p=0,087 ;$ Sibilos: $p=0,087 ;$ Dispneia: $p=0,018 ;$ Febre: $p=0,123$; Sono alterado: $p=0,040 ;$ Coriza: $p=0,360$

3A: Tosse: $p=0,020 ;$ Sibilos: $p=0,020 ;$ Dispneia: $p=0,760 ;$ Febre: $p=0,177 ;$ Sono alterado: $p=0,128 ;$ Coriza: $p=0,004$

F: Tosse: $p=0,128$; Sibilos: $p=0,136$; Dispneia: $p=0,146$; Febre: $p=0,018$; Sono alterado: $p=0,177$; Coriza: $p=0,562$ 
Em relação ao número de crises, duração da crise e internamento hospitalar, os grupos também mostraram distribuição homogênea em relação ao início do estudo. grupo que recebeu glutamina apresentou tendência estatística na redução do número de crises, no segundo mês de acompanhamento $(p=0,067)$, e redução no número de crises, no terceiro mês de acompanhamento $(p=0,049)$ (Tabela 2).

O grupo da glutamina também apresentou redução da duração de crise, no segundo e terceiro acompanhamento $(p=0,029, p=0,037)$, bem como na avaliação geral, após os quatro meses de suplementação $(p=<0,001)$. No entanto, notou-se que os melhores efeitos benéficos da glutamina ocorreram a partir do terceiro mês de suplementação (segundo acompanhamento). A ocorrência de internamento entre os grupos não apresentou diferença estatística significante (Tabela 2).

Em relação aos sintomas, no primeiro e segundo acompanhamento, observou-se melhora no sono, nos grupos da glutamina e do Lactobacillus. O grupo controle, embora sem diferença significante, apresentou maior prevalência de tosse, sibilo e coriza. No último acompanhamento, este grupo apresentou maior prevalência de febre $(p=0,018)$. Verificou-se que ocorreu menor prevalência de sintomas típicos de asma no grupo da glutamina, como: tosse, sibilo e coriza no terceiro mês de acompanhamento, e dispneia no segundo mês de acompanhamento (Figura 2).

Ao longo do estudo, os grupos de suplementação não apresentaram efeito colateral ou adversos da suplementação. Vale ressaltar que, no último mês de acompanhamento, ocorreu redução na periodicidade da utilização da glutamina em algumas crianças, o que pode ter influenciado na ocorrência das crises no último mês de estudo.

\section{DISCUSSÃO}

A asma resulta de fatores ambientais e genéticos que provocam interações entre células inflamatórias, mediadores e células estruturais que desencadeiam uma resposta inflamatória anormal nas vias aéreas, vindo a causar hiperreatividade das vias aéreas inferiores, obstrução do fluxo aéreo e sintomas como tosse, sibilos e dispneia ${ }^{3}$. Desta forma, percebe-se que o sistema imunológico tem papel importante nas características clínicas da asma.

Os resultados deste estudo indicam que a suplementação com glutamina pode atenuar os sintomas da asma. $\bigcirc$ possível mecanismo para este efeito pode ser justificado pela ação do aminoácido glutamina no aumento da produção de citocinas anti-inflamatórias, e de linfócitos T tipo 1, os quais tem função na regulação do sistema imune, pois in vitro estes foram capazes de inibir as citocinas com ação pró-inflamatória $^{10}$.
A suplementação da glutamina em ratos diminuiu a expressão da citocinas pró-inflamatórias, entre elas, interleucina-6 (IL-6) e fator de necrose tumoral alfa (TNFa), resultando na atenvação do processo inflamatório e síntese do óxido nítrico induzível (iNOS) ${ }^{11}$, vindo a destacar a efetividade da suplementação da glutamina. Uma vez que os indivíduos asmáticos têm maior quantidade de TNF- $\alpha$ que não asmáticos ${ }^{12}$ e a síntese do óxido nítrico induzível presente em células inflamatórias contribui para o estreitamento das vias aéreas em paciente asmáticos ${ }^{13}$, ressalta-se que a utilização deste imunomodulador pode auxiliar no tratamento da asma. No entanto, o presente estudo sugere que efeitos benéficos da glutamina na atenuação de sintomas de asma parecem ocorrer a partir do terceiro mês de utilização, indicando que a utilização a curto prazo talvez não seja capaz de auxiliar no tratamento da asma.

Além disso, em experimento com ratos induzidos às características clínicas de asma, que receberam a suplementação de glutamina, foi verificada inibição da ativação da fosfolipase citosólica A2 (cPLA2), bem como redução da atividade enzimática nas vias aéreas e da hiper-reatividade das vias aéreas na fase tardia ${ }^{14}$. Destaca-se que a ativação da cPLA2 tem papel importante na hiper-reatividade e inflamação das vias aéreas, características típicas da asma e doenças alérgicas, pois a TNF- $\alpha$ induz a formação de cPLA2, que libera ácido araquidônico, precursor de vários eicosanoides, como eucotrienos, prostaglandinas e fator ativador de plaquetas, que provocam a liberação de eosinófilos que levam à inflamação das vias áreas e broncoespasmos ${ }^{15}$.

Adicionalmente, a suplementação de glutamina também pode diminuir a quantidade de citocina tipo T2, que inibe a formação de CPLA2, o que levou a menor ou ausência de produção de muco em ratos ${ }^{14}$, podendo justificar o relato de menor coriza no grupo da glutamina no presente estudo. Destaca-se que um dos maiores fatores de mortalidade por asma é o excesso da produção de muco, que agrava as crises devido à obstrução das vias áreas, dificultando o fluxo de $a^{11}{ }^{16}$.

Os níveis baixos de glutamina no plasma de pacientes críticos também já estiveram associados ao maior risco de mortalidade ${ }^{17}$. Deste modo, também se nota a relevância da suplementação da glutamina para manter os níveis adequados deste aminoácido no organismo, para que o mesmo desempenhe suas funções no sistema imunológico, podendo evitar, assim, a ocorrência de crises de asma. Tal fato pode auxiliar na diminuição do risco de complicações e mortalidade em crianças com asma, bem como na redução do custo econômico despendidos com a doença.

A suplementação de glutamina em ratos também melhorou a oxigenação, funções mecânicas pulmonares, diminuiu recrutamento de neutrófilos e citocinas, o que 
resulta em menor inflamação das vias áreas e redução da lesão pulmonar ${ }^{18}$. Basicamente, o efeito clínico benéfico da suplementação de glutamina é explicado pela ação da glutamina no sistema imunológico, que diminui a expressão das citocinas pró-inflamatórias, TNF-a que resulta na diminuição da cPLA2, e eosinófilos, tendo como consequência a atenuação dos sintomas respiratórios e efeito protetor nas vias áreas.

Outra possível explicação para o efeito benéfico da glutamina seria que o aumento da citocina $\mathrm{Tl}$ tem ação na microbiota intestinal, levando ao aumento de linfócitos intraepiteliais intestinais que melhoram a integridade intestinal e barreira de proteção intestinal em estudos com ratos ${ }^{19}$. Isso poderia trazer benefícios para crianças asmáticas, pois - desenvolvimento da asma está associado à pequena variedade de microorganismos na microbiota intestinal nos primeiros anos de vida ${ }^{20}$.

Por outro lado, os Lactobacillus também têm efeito na função intestinal e na resposta imunológica' ${ }^{21}$. Entretanto, por meio de análise do material fecal, evidencia-se que o uso de antibiótico provoca a diminuição da variedade de microorganismo na microbiota intestinal ${ }^{22}$. Assim, o efeito do Lactobacillus na microbiota intestinal, que modularia a resposta imune para melhora da asma, pode ter sido prejudicado pelo uso concomitante de antibióticos neste grupo, visto que o grupo dos Lactobacillus não apresentou melhora significante na atenuação dos sintomas de asma. As hipóteses seriam que os Lactobacillus necessitam maior tempo de utilização para expressarem seus benefícios na modulação da microbiota intestinal e substituição da terapia medicamentosa de antibióticos para não provocar alterações na microbiota intestinal, a fim de não atrapaIhar o mecanismo dos Lactobacillus para atenuação dos sintomas clínicos da asma. Neste contexto, a suplementação de glutamina tem ação superior na atenuação dos sintomas de asma, devido a sua modulação do sistema imune, que traz benefícios ao sistema respiratório, que vão além do seu benefício na modulação da microbiota intestinal.

A relação entre o estado nutricional e a asma ainda é controversa. Em estudo de revisão, verifica-se que a obesidade possui associação com asma, os possíveis mecanismos são a resposta inflamatória nas vias aéreas, função mecânica pulmonar, resposta imunológica, questões hormonais e fatores genéticos. No entanto, a maioria dos estudos que relataram o fenótipo da obesidade na asma foi realizada em adultos, assim esta associação ainda é de aplicação questionável em crianças $^{23}$.

Apesar disso, crianças e adolescentes com obesidade têm maior prevalência de asma, maior número de hospitalizações e uso de corticoides ${ }^{24}$. Ainda, destaca-se que o uso de corticoides inalatórios estarem associado ao IMC elevado em crianças asmáticas ${ }^{25}$. No presente estudo, o grupo que recebeu glutamina apresentou melhora na classificação do estado nutricional pelo IMC/I após a suplementação, o que pode estar associado à atenuação dos sintomas clínicos da asma que levaram a menor utilização de medicamentos à base de corticoides, que podem influenciar no peso devido ao efeito colateral deste medicamento.

Algumas limitações do estudo devem ser mencionadas: o pequeno tamanho amostral; não cegamento para suplementação de glutamina e pool de Lactobacillus; o estado nutricional não foi critério para seleção da amostra, o que pode ser um viés do estudo, visto que tanto a obesidade quanto a desnutrição podem interferir na ocorrência da asma; a não avaliação da função intestinal e/ou microbiota intestinal, visto sua influência no sistema imunológico; por fim, não generalização dos dados, pois a população do estudo é específica. Como ponto positivo, ressalta-se que este estudo é o primeiro que avalia o efeito da suplementação da glutamina na atenuação dos sintomas clínicos da asma em humanos, utilizou o questionário ISAAC para diagnóstico dos sintomas da asma e controle da realização da suplementação da glutamina e Lactobacillus por meio do recebimento das embalagens vazias.

\section{CONCLUSÃO}

A suplementação de glutamina atenuou os sintomas típicos de asma, bem como reduziu a ocorrência e/ou duração das crises de asma, enquanto a utilização do pool de Lactobacillus não apresentou atenuação dos sintomas e crises de asma. Ambos os suplementos não apresentam efeitos colaterais e sua utilização foi segura. Nossos achados indicam que a glutamina pode ser uma nova estratégia para prevenção e controle da asma em crianças, visto que apresentou efeitos benéficos na melhora do estado clínico e estado nutricional, além de poder refletir em questões sociais e econômicas, pois diminuiu os episódios de crises, que pode levar a menor ausência escolar, diminuição de internamentos e redução dos gastos com medicamentos. Ensaios clínicos randomizados e cegos são necessários para confirmar os resultados do nosso estudo. Como perspectivas futuras de pesquisa, podemos destacar compreender se o efeito da glutamina se mantém a longo prazo ou se há necessidade da suplementação contínua para receber seus benefícios na atenuação dos sintomas das doenças respiratórias.

\section{AGRADECIMENTO}

À farmácia Trajano-Guarapuava/Paraná, pela doação da glutamina e pool de Lactobacillus. 


\section{REFERÊNCIAS}

1. Global Initiative for Asthma [Internet]. Global Initiative for Asthma. 2003. [citado 2015 out 17]. Disponível em: http://www. ginasthma.org/local/uploads/files/GINABurdenReport_1.pdf.

2. Subbarao P, Mandhane PJ, Sears MR. Asthma: epidemiology, etiology and risk factors. CMAJ. 2009;181(9):E181-90.

3. Holtzman MJ, Byersl DE, Alexander-Brett J, Wang X. The role of airway epithelial cells and innate immune cells in chronic respiratory disease. Nat Rev Immunol. 2014;14(10):686-98.

4. Sociedade Brasileira de Pneumologia e Tisiologia. IV diretrizes brasileiras para o manejo da asma. J Bras Pneumol. 2006;32(Suppl 7):S447-74.

5. Schmier JK, Manjunath R, Halpern MT, Jones ML, Thompson $\mathrm{K}$, Diette GB. The impact of inadequately controlled asthma in urban children on quality of life and productivity. Ann Allergy Asthma Immunol. 2007;98(3):245-51.

6. Mok EM, Hankard R. Glutamine supplementation in sick children: is it beneficial? J Nutr Metab. 2011;2001:(617597):1-41.

7. Mortaz E, Adcock IM, Folkerts G, Barnes PJ, Vos AP, Garssen J. Probiotics in the management of lung diseases. Mediators Inflamm. 2013;2013(751068):1-10.

8. Wandalsen NF, Gonzalez C, Wandalsen GF, Solé D. Avaliação de critérios para o diagnóstico de asma através de um questionário epidemiológico. J Bras Pneumol. 2009;35(3):199-205.

9. World Health Organization [Internet]. WHO Child Growth Standards: Length/height-for-age, weight-for-age, weight-for-leigth, weight-for-height and body mass index-for-age. Methods and development, 2006. [citado 2015 out 17]. Disponível em: http:// www.who.int/childgrowth/standards/Technical_report.pdf

10. Chang W, Yang KD, Shaio FM. Effect of glutamine on Th1 and Th2 cytokine responses of human peripheral blood mononuclear cells. Clin Immunol. 1999;93(3):294-301.

11. Singleton KD, Beckey VE, Wischmeyer PE. Glutamine prevents activation of NF-kB and stress kinase pathways, attenuates inflammatory cytokine release and prevents acute respiratory distress syndrome (ARDS) following sepsis. Shock. 2005;24(6):583-9.

12. Berry MA, Hargadon B, Shelley M, Parker D, Shaw DE, Green $\mathrm{RH}$, et al. Evidence of a role of tumor necrosis factor $\alpha$ in refractory asthma. N Engl J Med. 2006;354(7):697-708.
13. Sugiura H, Komaki Y, Koarai A, Ichinose M. Nitrative stress in refractory asthma. J Allergy Clin Immunol. 2008;121(2):355-60.

14. Ko HM, Kangw NI, Kimw YS, Leew YM, Jinz ZW, Jung YJ, et al. Glutamine preferentially inhibits T-helper type 2 cell-mediated airway inflammation and late airway hyperresponsiveness through the inhibition of cytosolic phospholipase A(2) activity in a murine asthma model. Clin Exp Allergy. 2008;38(2):357-64.

15. Choi IW, Sun-Kim, Kim YS, Ko HM, Im SY, Kim JH, et al. TNF-a induces the late-phase airway hyperresponsiveness and airway inflammation through cytosolic phospholipase A(2) activation. J Allergy Clin Immunol. 2005;116(3):537-43.

16. Rogers DF. Airway mucus hypersecretion in asthma: an undervalued pathology? Curr Opin Pharmacol. 2004;4(3):241-50.

17. Robas PC, Rooyackers O, Hebert C, Norberg A, Wernerman J. Glutamine and glutathione at ICU admission in relation to outcome. Clin Sci. 2012;122(12):591-7.

18. Lai CC, Liu WL, Chen CM. Glutamine attenuates acute lung injury caused by acid aspiration. Nutrients. 2014;6(8):3101-16.

19. Horio Y, Osawa S, Takagaki K, Hishida A, Furuta T, Ikuma M. Glutamine supplementation increases Th1-cytokine responses in murine intestinal intraepithelial lymphocytes. Cytokine. 2008;44(1):92-5.

20. Abrahamsson TR, Jakobsson HE, Andersson AF, Björkstén B, Engstrand L, Jenmalm MC. Low gut microbiota diversity in early infancy precedes asthma at school age. Clin Exp Allergy. 2014;44(6):842-50.

21. Saavedra JM. Use of probiotics in pediatrics: rationale, mechanisms of action, and practical aspects. Nutr Clin Pract. 2007;22(3):351-65.

22. Mikkelsen KH, Frost M, Bahl MI, Licht TR, Jensen US, Rosenberg J, et al. Effect of antibiotics on gut microbiota, gut hormones and glucose metabolism. PLoS One. 2015;10(11): e0142352.

23. Raj D, Kabra SK, Lodha R. Childhood obesity and risk of allergy or asthma. Immunol Allergy Clin North Am. 2014;34(4):753-65.

24. Black MH, Smith N, Porter AH, Jacobsen SJ, Koebnick C. Higher prevalence of obesity among children with asthma. Obesity (Silver Spring). 2012;20(5):1041-7.

25. Van Gent R, van der Ent CK, Rovers MM, Kimpen JL, van EssenZandvliet LE, Meer G. Excessive body weight is associated with additional loss of quality of life in children with asthma. J Allergy Clin Immunol. 2007;119(3):591-6.

\section{Local de realização do estudo: Universidade Estadual do Centro-Oeste, Guarapuava, PR, Brasil.}

Conflito de interesse: Os autores declaram não haver.

Foi apresentado como trabalho de destaque no XXIII Congresso Brasileiro de Nutrição Parenteral e Enteral, entre 20 a 23 de outubro de 2019, em Foz do Iguaçu-PR. 